\title{
Ejercicio del poder desde la perspectiva de las necesidades de los directivos en organizaciones públicas ${ }^{1}$ \\ Exercise of power from the perspective of the needs of directors in public organizations
}

DOI: http://dx.doi.org/10.17981/econcuc.38.2.2017.03

Recibido: may 23/2017 Devuelto para revisión: jul 5/17 Aceptado: oct 24/2017

\author{
Marlén Suárez Pineda ${ }^{2}$ \\ Sandra Milena Zambrano Vargas ${ }^{3}$ \\ Jenny Edith Parada Camargo ${ }^{4}$
}

\begin{abstract}
Resumen
El presente artículo expone una experiencia investigativa cuyo propósito se centró en la aproximación al fenómeno del ejercicio del poder en organizaciones públicas para caracterizar la naturaleza del mismo desde la perspectiva de las necesidades de quien ejerce autoridad racional dentro de la estructura organizacional. El estudio sigue un enfoque mixto, de tipo descriptivo, apoyado en las herramientas de escala Likert y entrevista semiestructurada. Los resultados retratan un directivo cuya interacción social es mayormente dominada por la necesidad de afiliación y que es percibido por los colaboradores como un líder de referencia.
\end{abstract}

Palabras clave: Estilos de poder, necesidades de los directivos, poder legítimo, poder de referencia, organizaciones públicas.

\begin{abstract}
This article presents a research experience which purpose was focused on the approach to the phenomenon of the exercise of power in public organizations, to characterize the nature of the same from the perspective of the needs of those who exercise rational authority within the organizational structure. The study follows a mixed approach, of descriptive type, supported by Likert scale tools and semi-structured interviews. The results portray a manager whose social interaction is largely dominated by the need for affiliation and whose is perceived by the collaborators as a reference leader.
\end{abstract}

Keywords: Styles of power, managerial needs, legitimate power, power of reference, public organizations.

\footnotetext{
${ }^{1}$ Artículo científico como resultado del proyecto de investigación "Estilos de poder y las subyacentes necesidades de los directivos en organizaciones públicas de Boyacá" realizado por el grupo de investigación IDEAS, adscrito a la Escuela de Administración de Empresas de la Universidad Pedagógica y Tecnológica de Colombia.

${ }^{2}$ Magíster en Administración; doctoranda en Administración de la Universidad Autónoma de Querétaro; docente de la Escuela de Administración de Empresas de la UPTC de Tunja; directora del grupo de investigación IDEAS. Correo: marlen. suarez@uptc.edu.co

${ }^{3}$ Magíster en Administración de la Universidad Nacional de Colombia; doctoranda en Administración de la Universidad Autónoma de Querétaro; integrante del grupo de investigación IDEAS; docente de la Escuela de Administración de Empresas de la UPTC, Tunja. Correo: sandra.zambrano@uptc.edu.co.

${ }^{4}$ Magíster en Dirección y Administración de Empresas; integrante del grupo de investigación Poder, perteneciente a la Escuela de Administración de Empresas de la UPTC Tunja; docente de la Escuela de Administración de Empresas de la UPTC Tunja. Correo: jenny.parada@uptc.edu.co.
} 


\section{Introducción}

Las discusiones en el campo de la teoría organizacional acerca del ejercicio del poder han mantenido un carácter racional estructural, siguiendo la visión weberiana, según la cual las personas ejercen poder cuando influyen en el comportamiento de otros, haciendo que éstos hagan lo que en ausencia de tal influencia no harían. La organización, por su naturaleza de sistema social, es el escenario típico de interacción que expone las complejidades propias del fenómeno del poder. Reconocer e interpretar significados y representaciones entre los actores en las organizaciones, particularmente dentro de la relación jefe-colaboradores, debe permitir hacer aportes a la explicación de los comportamientos intraorganizacionales $\mathrm{y}$, asimismo, debe sumar como evidencia empírica de las proposiciones formuladas por los teóricos elegidos. En esta oportunidad, las explicaciones se pretenden para el ámbito de organizaciones de carácter público en un espacio territorial. La investigación no tiene pretensiones de generalización por considerar las singularidades asociadas a la cultura tanto interna como del ambiente de las entidades observadas.

\section{Fundamentos teóricos sobre el poder}

Resulta fundamental entonces empezar por comprender el concepto de poder, el cual se puede abordar a partir de contribuciones del sociólogo Weber (2012), quien expresa que el poder se muestra en la probabilidad de imponer la voluntad de una persona, dentro de una relación social, aun contra toda resistencia del otro, o de los otros, y cualquiera sea el fundamento de esa posibilidad. García (2009) pretende mostrar el poder como un referente para encontrar explicación a la conducta de los colaboradores en las empresas y permite comparar lo que desde la administración se ha conocido como el ejercicio del poder en las organizaciones. Explica el poder como la acción que se ejerce sobre los seres humanos por otros seres humanos, además, menciona que las ciencias humanas fundamentales identificaron hace tiempo que el ser humano es un ser de subjetividad y de símbolos; sus comportamientos, su sentido de pertenencia frente a otros actores, sus promesas, sus afectos, etc. pasan por el ejercicio del poder. Foucault (1988) muestra unas necesidades conceptuales en la cuestión del sujeto y la relación de poder:

Así, el poder designa relaciones entre "parejas" en un conjunto de acciones que se inducen y forman una sucesión; las disciplinas muestran el modo como pueden articularse los sistemas de finalidad objetiva, los de comunicación y los de poder; el Gobierno designa el modo de dirigir la conducta de individuos o grupos; y la estrategia se utiliza en tres sentidos: la racionalidad empleada para alcanzar un objetivo, la manera en la que se trata de tener ventaja sobre el otro y los medios destinados a obtener la victoria.

De igual forma, Foucault (1992) habla del poder desde el Estado, el cual sin duda permite el ejercicio del mismo. Sin embargo, explora algunas incógnitas como ¿quién ejerce el poder? y ¿dónde lo ejerce?, porque se sabe quién lo explota y a dónde van sus resultados y, además, se sabe que no propiamente los dirigentes tienen el poder. Por tanto, es importante conocer hasta qué instancias se ejerce y por cuáles conexiones. En todo caso, para Foucault, en todo lugar en donde exista poder, éste se ejerce por alguien.

Mintzberg (1989) también habla de los factores de poder, como el control externo de la organización, las necesidades personales de sus miembros y la moda del día; a partir de esto describe las siguientes hipótesis: cuanto mayor el control externo de la organización, más centralizada y formalizada es su estructura; y las necesidades de poder de los miembros tienden a generar estructuras que son excesivamente centralizadas. 
Pelegrí (2004) muestra las relaciones de poder y el trabajo social; dice que es precisamente por esta posición diferenciada por la que un cliente acude a un profesional: el profesional está en disposición de ayudarle y se produce, de hecho, una relación de ayuda que resulta efectiva para el cliente. De la Rosa (2007) enfatiza en la comprensión de las relaciones de poder existentes entre las personas, en sus palabras, el poder aparece como racionalmente instrumental, sin embargo, las relaciones de poder no necesariamente se conciben desde esa racionalidad, ya que, en el diario vivir, esas relaciones pueden estar permeadas por las lógicas de acción conscientes o inconscientes de los individuos dentro de la organización.

Por su parte, Arana y Foutel (2007) afirman que el macropoder se refiere al uso del poder por parte de la organización, en cooperación o conflicto con otras, reflejando el grado de interdependencia entre ella y su entorno. Con la perspectiva del macropoder, la vinculación consiste en manejar, primero, la demanda de estos actores y, segundo, en utilizarlos selectivamente en beneficio de la organización. Más allá de las múltiples organizaciones que imponen marco regulatorio y proveen insumos o recursos, deben destacarse aquí aquellas que originan en los participantes conflictos de roles o lealtades. Por su parte Weber (1997), afirma en su texto que la mayor influencia efectiva en lo político no proviene fundamentalmente de los factores económicos; su origen hay que buscarlo en sentimientos de prestigio frecuentemente muy arraigados en las masas pequeñas burguesas pertenecientes a formaciones políticas que han sido los poseedores del poder.

Finalmente, Altomare (2010) busca reconstruir los tres modos de orientación de la acción de la sociología de Max Weber referentes a la acción social, relación social y el orden legítimo. La acción social hace referencia a la comprensión y sentido, la relación social sucede cuando se combinan las acciones para que fluyan más rápido y se da una interacción entre las acciones y el orden legítimo. Weber (2012) diferencia un tipo de regularidad en la relación social en la que los participantes se orientan por la representación de un orden legítimo. "No son otra cosa que desarrollos y entrelazamientos de acciones específicas de personas individuales" (Weber, 2012, p. 12).

\section{Poder social y liderazgo}

La teoría del poder social, según French y Raven (1982), muestra cinco fuentes de poder que son: el poder coercitivo, el poder por recompensa, el poder legítimo, el poder del experto y el poder referente. En cuanto al poder coercitivo, un agente A impone su voluntad a través de amenazas o castigos a un agente B. El poder por recompensa utiliza recursos que $\mathrm{B}$ desea y valora positivamente; genera un aumento de la atracción hacia el poder y disminuye la resistencia. El poder legítimo es aquel que por un conjunto de normas establecidas y conocidas de antemano impone ese poder sin discusión por su posición (organización, familia, sociedad), definiéndose como autoridad (Weber, 2007). El poder del experto lo ejerce quien es reconocido por el grado de conocimientos, habilidades, autoridad que posee sobre un tema; en éste, el dominador es apoyado por los dominados gracias a las habilidades, destrezas, conocimientos por una disciplina, ocupación o labor. Se pueden dar relaciones de seguridad y competencia (Kim, Pinkley y Fragale, 2005). Y, por último, el poder referente se ejerce con base en los sentimientos de respeto, admiración, confianza y lealtad hacia quien ejerce el poder.

Contreras y Castro (2013) analizan los procesos de liderazgo y poder a partir de interacciones que se dan entre las personas que conforman una organización, afirmando, como conclusión, que el liderazgo y el poder movilizan a la organización y pueden ocasionar desequilibrios productivos, los cuales favorecen el desarrollo de las organizaciones 
y su perdurabilidad. Los resultados fueron obtenidos a partir de comprender la influencia que tienen los seguidores/colaboradores sobre el líder y sobre los procesos de liderazgo, y, así mismo, comprender que el poder y el liderazgo no son fenómenos aislados, sino que cooperan para que las organizaciones sean cada vez más competitivas.

Kadi y Acevedo (2014) hablan de los conceptos de poder y autoridad a partir de la influencia para introducir cambios en el comportamiento de las personas. De esta forma, el líder sería un factor clave en cualquier ámbito, ya que puede ayudar a orientar a un colectivo hacia el logro de los objetivos propuestos.

Cruz (2013) se refiere a la dominación que se ejerce desde una posición de superioridad establecida formalmente en la organización a través de del sistema legal imperante: "la dominación que se ejerce a partir de este sistema de dominio es impersonal debido a que responde a un mecanismo de participación sustentado en las normas, y por lo tanto, quien ejerce la dominación no requiere la legalidad imperante, con lo cual el elemento coercitivo de dominio se convierte en un mecanismo latente de control" (p. 55). De igual manera, existen organizaciones, en su mayoría públicas, que, a pesar de tener metas claras y los medios para lograr buenos niveles de eficiencia, no pueden alcanzarlos debido a la atención de las racionalidades individuales de los grupos de poder (Vargas, 2014).

Serna (2013) afirma que el "desarrollo de organizaciones empresariales complejas genera nuevas fuentes de poder vinculadas a la gestión empresarial, al tradicional empresario propietario, se suma la figura de la alta gerencia en la cabeza de las empresas" (p. 94). Los cambios que ha tenido el capitalismo han traído consigo un cambio organizacional, en donde se tienen diferentes herramientas de gestión y una estructura flexible que da autoridad menos firme en la relación de la dirección, la alta gerencia y el accionista con los mandos medios y trabajadores.

Tirado (2015) nos dice que el poder se diferencia a partir de la cantidad de asociados o dependientes de la organización y de sus redes institucionales, que permiten la flexibilidad y el dinamismo entre los actores, sin embargo, cada institución tiene una lógica propia en donde se pone en evidencia que puede haber contextos iguales pero que despliegan especificaciones diferentes del poder. "Los actores controladores de muchos recursos (de toda índole) tienden a desarrollar redes de relaciones en las que son muy activos, de modo que constituyen un repertorio de relaciones/acciones que les permiten usar diferentes mecanismos de poder para incidir en la acción de otros actores, aunque portan la lógica institucional" (p. 335).

Fuentes (2014) cita a Foucault (1988) sobre la influencia de los grupos de interés y su relación con la organización que, para el caso de estudio del autor, es Ecopetrol, donde "en estas relaciones, unos actores actúan sobre las acciones de los otros; y en una cotidianidad como la examinada aquí, se traduce en la transformación de los individuos en sujetos de poder. Esta complejidad no requiere ser solucionada sino problematizada, ejercicio que inicia con la identificación de formas de resistencia contra los diferentes tipos de poder."

Molina (2012) dispone que una de las fuentes de poder importante es la comunicación; es una alternativa valiosa para alcanzar objetivos en donde se le da la oportunidad y la responsabilidad para identificar problemas y resolverlos. Este poder requiere de una mayor cantidad de participación y aprendizaje que el contar solamente con una persona encargada de tomar decisiones. Ampliando lo anterior, García (2014) indica que es necesario que el líder o el directivo esté presente dentro de la organización como 
una fuente de poder, es decir, tener presencia activa para el logro de los objetivos; cuando no se tiene un individuo al mando o todo se hace por la vía de los medios de comunicación, puede suceder que otro tome la decisión de hacerlo, aunque no esté dentro de sus funciones o el directivo piense que no es necesario que esté presente. En el mismo sentido, Mariño (2014) hace énfasis en la comunicación como un mecanismo de poder que puede ser muy efectivo, pues le permite lograr los objetivos propuestos, como resultado del ejercicio del poder, y, de esta manera, es posible el uso del discurso y la comunicación como una forma de generar lazos de cooperación entre los miembros de las organizaciones y entre los diferentes niveles jerárquicos existentes en las mismas.

Yela e Hidalgo (2010), en su trabajo, analizan el poder desde la perspectiva de Michel Foucault. En un principio, se tomó el poder como el esfuerzo de establecer control y disciplina, que diera orden, obediencia y regularizara a los trabajadores, éste se asoció de forma negativa con la idea de represión y prohibición, llamado también poder jurídico. Por eta razón, nace un poder de estrategia, en donde se trasforma el poder tradicional por el que se da a partir de la objetividad del individuo a través de la vida cotidiana y su propia singularidad: el poder instrumentalista. Éste se emplea como herramienta de gestión empresarial, un recurso frente al cual los directivos dan uso efectivo y estratégico y con el que se obtengan beneficios personales y el logro de los objetivos organizacionales, que utilice aspectos como la delegación de poder a los niveles "bajos" de la pirámide jerárquica y en donde haya una mayor participación de los colaboradores en la toma de decisiones de la organización.

Murillo (2009, p. 41) plantea que, para Weber, existen tres tipos de sociedad, las cuales dan origen a tres tipos de dominación: "dominación tradicional, basada en las costumbres y prácticas sociales de las diferentes comunidades; dominación carismática, basada en las características intrínsecas de los individuos, asociadas a su forma de ser y de actuar; y la dominación tradicional o legal, estructurada a partir de elementos racionales y objetivos, que pretende privilegiar algunas características 'impersonales' de carácter abstracto y que desde el punto de vista metodológico se establece como un 'tipo ideal' de construcción de categorías sociales (...)". También afirma que en Latinoamérica se ve la economía como fuente de poder y autoridad, y esto en sociología, como cohesión social.

Para García (2009), el ejercicio del poder en las organizaciones, desde la práctica, hace que se pueda poner en evidencia de forma real la conducta y el poder como fuerza de cohesión de los colaboradores en el entorno que los rodea. Partiendo de ello, se habla de poder desde diferentes autores, como por ejemplo Freud (1976), quien reconoce la resistencia como parte del poder en donde se da una relación del súper yo y el yo del individuo, para lo cual no es evidente la expresión de resistencia en las relaciones de poder en un grupo; y Russell (1968) menciona dos tipos de poder: el tradicional, que se identifica por las costumbres y creencias, y el poder adquirido, el cual nace de la necesidad, como fuente de poder revolucionario en donde se ve la expresión objetiva de la resistencia.

\section{Perspectivas del poder}

Penaglia (2012) afirma que el poder posee dos perspectivas que pueden ser excluyentes entre sí. El poder se construye a través de relaciones sociales y acciones en conjunto, pero también se construye en el desarrollo de capacidades individuales. El poder entonces tiene un paradigma social y paradigma individual, a esto se le se denomina la paradoja del poder, y nace para que los individuos potencien sus capacidades y alcancen sus objetivos de manera colectiva, lo cual implica ceder niveles de autonomía y libertad en pro de intereses comunes. 
Rodríguez (2015) afirma que las relaciones de poder se dan a partir de las emociones que experimenta el ser humano cuando ejerce una determinada actividad; está condicionada por el grado jerárquico y por las condiciones de desigualdad. Lo anterior hace que sea más evidente la toma de decisiones que afecta a un determinado grupo poblacional, ya sea por su grado de riqueza, escolaridad o por el ejercicio de dominación que se ha desarrollado por la tradición del poder que se presenta en torno al status, las creencias, los intereses, preferencias y deseos. Para el caso analizado por Abernethy y Vagnoni (2004), la autoridad formal está representada por la asignación del derecho a la toma de decisiones, que, por lo general, se relaciona con la posición de quien ejerce el poder en una jerarquía clasificada.

La estrategia también es utilizada como fuente de poder para que un individuo actúe como desea el sujeto que ejerce el poder, de tal forma que éste proceda a la luz de sus acciones y actúa como reacción de la depredación organizacional, la cual se da a partir de la resistencia natural que existe al poder de otros actores, pues quiere controlar parte de la organización para adquirir dicho poder (Castro y Noguera, 2014; Contreras y Castro, 2013). Así mismo, Sanabria, Trujillo y Guzmán (2008) afirman que "la base del poder dentro de una organización se deriva de la habilidad de una persona o de un grupo de personas para tomar o no acciones que son deseadas por otros".

Cuando se habla de las necesidades de las personas, hay que referirse a McClelland (1989), quien sostuvo que todos los seres humanos poseen unas necesidades comunes; en primer lugar, tiene unas necesidades de logro, referidas al esfuerzo por sobresalir y la lucha por el éxito; en segundo lugar, tiene unas necesidades de poder, referidas al deseo de conseguir que las demás personas se comporten en una manera que no lo harían, es decir, se analiza el deseo de tener impacto, de influir y controlar a los demás; por último, unas necesidades de afiliación, entendidas como el deseo de relacionarse con las demás personas, es decir, de entablar relaciones interpersonales amistosas y cercanas con los demás integrantes de una organización.

La revisión de literatura y las expectativas del investigador condujeron a la selección de la propuesta teórica de French y Raven (1982) para caracterizar el ejercicio directivo desde las fuentes de poder que mejor identifican a los sujetos con designaciones de autoridad en la estructura organizacional. Del mismo modo, se selecciona la teoría de David McClelland (1989) sobre las necesidades del ser humano, para orientar con una mayor aproximación hacia la definición del papel del directivo desde las necesidades que subyacen a las formas de influir en el equipo colaborador.

\section{Metodología}

La población objeto de observación se define como las organizaciones de carácter estatal con ámbito de operación en el departamento de Boyacá, Colombia. Se seleccionó una muestra por conveniencia de 20 organizaciones, prefiriendo la participación de los sectores de educación, salud y organismos administrativos de nivel territorial. Después de un proceso de sensibilización sobre los propósitos del estudio y de la gestión del conocimiento informado, se obtuvieron 12 organizaciones que aceptaron participar.

En cada una de las organizaciones se seleccionó un directivo, bajo el criterio de que ocupara un cargo ubicado dentro de los órganos de decisión de la entidad y que tuviera autoridad y control formal dentro de la estructura. Con cada uno de estos doce directivos se desarrolló una entrevista semiestructurada siguiendo la guía diseñada para promover la identificación de las necesidades referidas en la teoría. 
Del mismo modo, en cada organización se aplicaron cuestionarios tipo Likert a los colaboradores de los directivos entrevistados, lográndose la aplicación de 54 cuestionarios. El cuestionario fue diseñado previendo cinco dimensiones de análisis correspondientes a las cinco fuentes de poder referidas por French y Raven (1982).

El análisis de los datos se hizo en congruencia con la herramienta de recolección, así, las entrevistas se analizaron definiendo tres categorías de datos, según las necesidades de McClelland (1989), interpretando representaciones expuestas en el discurso. Por su parte, los cuestionarios fueron revisados con herramientas de tipo estadístico que permitieran considerar percepciones traducidas en expresiones cuantitativas.

\section{Resultados y discusión}

\section{Análisis cuantitativo:}

Los estilos de poder según percepciones de los subordinados o colaboradores

Se precisa previamente que el subordinado, para los fines de este estudio, sea el actor sobre quien recae la influencia de aquel que ejerce poder o dominación y que, simultáneamente, está ubicado en posición de autoridad dentro de la estructura organizacional.

La sistematización y análisis del cuestionario tipo Likert aplicado a funcionarios públicos en su papel de colaboradores, receptores de la influencia de un jefe, permite hallar un promedio de valoración, en una escala de 1 a 5 , para el grupo de preguntas ubicadas en cada una de las cinco categorías, definidas como estilos de poder: legítimo, coercitivo, de recompensa, de referencia y de experto. De este modo, se obtienen los siguientes valores promedio por categoría:
Tabla 1

Promedio de la valoración de los estilos de poder percibidos por los subordinados.

\begin{tabular}{cc}
\hline $\begin{array}{c}\text { Estilo de } \\
\text { poder }\end{array}$ & $\begin{array}{c}\text { Promedio de valoración } \\
\text { Percepción nunca: } \mathbf{1} \\
\text { Percepción siempre: } \mathbf{5}\end{array}$ \\
\hline Referencia & 3,99 \\
Legítimo & 3,97 \\
Experto & 3,92 \\
Recompensa & 2,88 \\
Coercitivo & 2,37 \\
\hline
\end{tabular}

Fuente: Elaboración propia (2017).

Desde las representaciones de los consultados, que cumplen roles de subordinación en la estructura organizacional prescrita, el poder mayormente percibido es el de referencia, lo cual hace suponer, de acuerdo con las orientaciones teóricas dominantes en este estudio, que la influencia lograda por los jefes sobre sus colaboradores está derivada particularmente de la imagen que los primeros son capaces de proyectar sobre los segundos, los cuales son percibidos como jefes comprometidos, investidos de valores y dignos de confianza. Se espera, en consecuencia, que la respuesta más probable de sus subordinados sea el de compromiso, antes que acatamiento o resistencia. Para alcanzar la medición de la percepción con respecto a esta categoría de poder se consultaron aspectos relacionados con la admiración al jefe, el deseo de emularle, la identificación con las formas de actuar, el respeto y la confianza que inspira y la apreciación sobre su honestidad.

Un enunciado inductivo defendido por esos resultados puede tratarse de que los funcionarios públicos del departamento de Boyacá reconocen tener jefes a quienes desean acompañar en sus propósitos, por la integridad que representan y la admiración que les merecen. En consecuencia, puede inducirse que el compromiso con 
los fines de la organización es una de las respuestas que más se repiten en el comportamiento de los subordinados.

Después del poder de referencia, y con un margen muy escaso de diferencia, los colaboradores perciben a sus jefes como aquellos que ejercen un poder legítimo. En esta categoría se ubican entre otros los cuestionamientos que se aproximan a la medición de: la importancia del jefe en la jerarquía organizacional, el merecimiento de obediencia por su naturaleza de jefe, el reconocimiento de autoridad en cabeza del jefe y la obediencia a las indicaciones del jefe. Llama la atención que los consultados reconocen (con valoración de siempre) que su jefe es una persona "más importante" que ellos dentro de la organización y que las indicaciones proferidas por él (el jefe) deben ser atendidas porque es el jefe y representa autoridad.

De acuerdo con este hallazgo, puede construirse una proposición de tipo inductivo que caracteriza al funcionario público del departamento como un respetuoso de las prescripciones normativas, y en ese orden, todo aquel que posea el mandato está justificado para ejercer potestad. Evidencias empíricas respaldan la tesis de que la respuesta más probable de los subordinados ante este estilo de poder es el acatamiento, lo cual llama la atención acerca de la responsabilidad sobre los resultados que éstos asumen y que, a la postre, resulta amenazante para el desempeño de las organizaciones.

En un tercer orden, las percepciones se ubican en el poder de experto, lo cual hace comprender que el jefe es seguido y apoyado gracias a sus habilidades, destrezas y conocimientos, aspectos que confieren seguridad a lo subordinados con respecto a las posibilidades de éxito en el logro de los propósitos organizacionales. En esta categoría, las indagaciones se orientan a identificar cuál es el grado de admiración que el subordinado tiene de su jefe por los conocimientos que éste posee, cuál es nivel de confianza en el jefe por conocer de los asuntos que le competen y cuánto se justifica el seguimiento al jefe por las habilidades, conocimientos y experiencia que demuestra.

Las preguntas ubicadas en las categorías de recompensa y coercitivo fueron valoradas por debajo de 3 en la escala de 1 a 5 utilizada en la sistematización de los datos. Se interpreta que estos dos tipos de poder son apreciados escasamente. Los funcionarios no se perciben influenciados por jefes que despiertan tensión y poca confianza, no se sienten acatando órdenes por temor a recibir represalias o por la expectativa de ser recompensados. Los resultados no indican que estén ausentes estas dos modalidades de poder, pero sí señalan que son escasos.

En el caso del estilo coercitivo, el instrumento pretendía una aproximación para conocer el grado en el que el subordinado está dispuesto a dar obediencia por temor a revanchas del jefe, cuánto siente que no le está permitido mostrar desacuerdos y cuánta amenaza percibe en las actuaciones y el discurso del jefe. Por su parte, en la categoría de recompensa, se incorporaron interrogantes conducentes a develar en qué nivel los funcionarios perciben que su jefe influye para asignar incentivos y si los asigna con criterios asociados al acatamiento de sus orientaciones, en qué grado los funcionarios desarrollan acciones condicionadas por una retribución y cuál es el grado de asociación entre las condiciones laborales de las personas y las afinidades sociales con los superiores.

Una proposición congruente con los resultados en esta categoría, puede trazar- 
se en la línea de que los funcionarios públicos de la región no perciben jefes que les coarten su autonomía. El desempeño del personal no está inspirado en el temor ni en la pretensión de favores, mas sí lo está en el respaldo a líderes íntegros y la obediencia debida al jefe legítimo.

\section{Análisis cualitativo:}

Las necesidades de los directivos expresadas en su ejercicio del poder.

Una segunda parte del análisis de la información es la identificación de las motivaciones y necesidades de los directivos tras la pesquisa de cuál de las necesidades de la teoría seleccionada es la que más se manifiesta en la interacción del directivo con su equipo de trabajo. La interpretación de las respuestas ante la indagación sobre la mejor experiencia de ser jefe, permite una interpretación a favor de que la necesidad de afiliación es la que se impone, respaldada en la satisfacción expresada por haber brindado ayuda a otros.

En referencia con la expectativa que el jefe tiene de sus colaboradores, se interpreta que coexiste la necesidad de poder con la necesidad de logro, se expresa el deseo de controlar a las personas conjuntamente con la presión por alcanzar las metas a través de ellas.

Indagando sobre las metas como jefes, se encuentra que al mayor número de directivos les asiste gran compromiso con metas organizacionales. Un grupo menor de directivos reconoce metas relacionadas con la calidad de vida de los colaboradores. Estos hallazgos se traducen en que las necesidades de logro y de afiliación son dominantes entre los directivos. En relación con las metas de orden personal se expone una tendencia a buscar metas de formación intelectual y se repite la pretensión de querer hacer por otros.
La entrevista estimuló la ocupación en otros asuntos como: los principales logros como jefe, dificultades enfrentadas en el desarrollo del rol de jefe, estrategias utilizadas para ganar respuestas oportunas de los colaboradores, percepción sobre cómo le calificarían los colaboradores, estrategias para mantener el compromiso de los colaboradores, resolución de conflictos, aspectos personales y profesionales que desea mejorar, cualidades personales y profesionales que influyen positiva y negativamente en las relaciones con los colaboradores. Los consultados dieron respuestas que, interpretadas a la luz de la teoría de las necesidades de McClelland (1982), permiten encontrar que hay una necesidad dominante manifestada en el ejercicio del poder de los directivos: la necesidad de afiliación. Un enunciado que puede construirse es el de que las personas que ejercen cargos directivos en las organizaciones públicas del departamento experimentan la necesidad de afiliación antes que la de poder y logro. Es recurrente encontrar que en relación con la mejor experiencia vivida como jefe, la persona responde "poder ayudar a la gente", "apoyar al subalterno", "el trabajo en equipo que he podido lograr". En el mismo sentido, los jefes esperan de sus colaboradores que "no tengan miedo, se preparen y sean proactivos" y "que logren sus objetivos". La estrategia utilizada para que los colaboradores atiendan oportunamente lo solicitado está relacionada con el diálogo amable con ellos, el otorgamiento de confianza y el trato respetuoso.

En la tabla 2 se resumen las necesidades a las que corresponden la mayoría de las representaciones exteriorizadas por los directivos consultados por cada variable utilizada. La necesidad que se cita primero en cada variable se refiere a la que tiene mayor presencia. 
Tabla 2

Necesidades del directivo con mayor manifestación en las variables.

\begin{tabular}{|c|c|}
\hline Variable & $\begin{array}{l}\text { Necesidad del directivo } \\
\text { mayormente manifestada }\end{array}$ \\
\hline Experiencias representativas en el ejercicio como jefe & $\begin{array}{l}\text { Necesidad de afiliación } \\
\text { Necesidad de logro }\end{array}$ \\
\hline Expectativa acerca de los colaboradores & $\begin{array}{l}\text { Necesidad de poder } \\
\text { Necesidad de logro }\end{array}$ \\
\hline Metas como jefe & $\begin{array}{l}\text { Necesidad de afiliación } \\
\text { Necesidad de logro }\end{array}$ \\
\hline Logros como jefe & $\begin{array}{l}\text { Necesidad de afiliación } \\
\text { Necesidad de logro }\end{array}$ \\
\hline Dificultades enfrentadas en el rol de jefe & Necesidad de afiliación \\
\hline Estrategias para ganar respuestas de los colaboradores & $\begin{array}{l}\text { Necesidad de afiliación } \\
\text { Necesidad de poder }\end{array}$ \\
\hline Calificación que percibe de parte de los colaboradores & $\begin{array}{l}\text { Necesidad de afiliación } \\
\text { Necesidad de logro }\end{array}$ \\
\hline $\begin{array}{l}\text { Estrategia para mantener compromiso de } \\
\text { colaboradores }\end{array}$ & Necesidad de afiliación \\
\hline Estrategias para resolución de conflictos & $\begin{array}{l}\text { Necesidad de afiliación } \\
\text { Necesidad de poder }\end{array}$ \\
\hline Aspectos personales/profesionales para mejorar & $\begin{array}{l}\text { Necesidad de afiliación } \\
\text { Necesidad de poder }\end{array}$ \\
\hline $\begin{array}{l}\text { Cualidades personales y profesionales que influyen en } \\
\text { relaciones con colaboradores }\end{array}$ & $\begin{array}{l}\text { Necesidad de poder } \\
\text { Necesidad de afiliación }\end{array}$ \\
\hline
\end{tabular}

Fuente: elaboración propia (2017).

Se refleja que las tres necesidades son experimentadas por los directivos, pero es la de afiliación la que se muestra en primer orden en nueve de las once variables consideradas. La necesidad de poder está reflejada en cinco de las once variables, en esa misma medida está presente la necesidad de logro. Desde la orientación de la teoría utilizada se infiere que al ejercicio del poder por parte de los directivos subyace una necesidad de relacionarse con las demás personas, de mantener relaciones amistosas y construir interacciones de apoyo mutuo. Las necesidades de tener éxito en los propósitos y de controlar a los demás son experimentadas en menor medida, es decir, gobiernan menos el comportamiento del directivo.

\section{Conclusiones y recomendaciones}

Las personas en las organizaciones obran de acuerdo con sus motivaciones y responden a los estímulos del entorno según esas motivaciones. Los directivos de las organizaciones públicas del departamento, de acuerdo con los resultados, se ven motivados por afianzar relaciones amistosas con su equipo de trabajo, los estimula la interacción social en donde haya apoyo mutuo, sus metas están traspasadas por el interés de ser aceptado y apreciado por los demás. Se esperaría entonces que las organizaciones, en promedio, mostraran una tendencia a gestionarse en un ambiente de empoderamiento, dominio propio, autonomía y trabajo de equipo. 
La necesidad de poder y de logro está también presente como una motivación en la cotidianidad de los directivos expresándose en menor medida. Por su parte, las representaciones de los subordinados se reúnen alrededor de que su jefe tiene influencia derivada de su imagen de persona íntegra y digna de confianza. En un segundo orden, las percepciones muestran que los jefes tienen capacidad de influencia derivada del cargo, lo cual supone fuerte apego a normas y jerarquías preestablecidas. La influencia que emerge del conocimiento y la experiencia también es percibida entre las tres fuentes dominantes. La capacidad para prodigar recompensas o castigos a los subordinados son las que están menos presentes en las representaciones de los consultados, lo cual abre camino para el enunciado de que son escasos los ambientes de trabajo intimidatorios y de negociación de compensaciones por seguir las orientaciones del jefe. De acuerdo con los resultados, es de esperarse que el perfil de directivos de mayor presencia en las organizaciones públicas del departamento los empuje hacia la construcción de visiones compartidas que deben reflejar, en el tiempo, el fortalecimiento de su competitividad.

El carisma y la legitimidad se reflejan como la fuente de donde deriva el poder que los jefes ejercen sobre sus equipos de colaboradores, lo cual es coherente con el tipo de estructuras organizacionales, de corte rígido, altamente jerarquizadas y centralizadas, con dificultades para asumir los cambios, donde la gestión atiende más a lo prescrito en el diseño organizacional que a los propósitos estratégicos de la organización. Los resultados aportan a la comprensión del fenómeno del poder en el tipo de organización y el territorio del estudio.

\section{Referencias}

Abernethy, M. y Vagnoni, E. (2004). Power, organization design and managerial behaviour. Accounting, Organizations and Society, 29, 207-225.
Altomare, M. (2010). Las dimensiones del sentido en la teoría social de Max Weber: acción social, relación social y orden legítimo. Perspectivas en psicología, 7, 40-44.

Arana, M. y Foutel, M. (2007). Poder y gobernabilidad. VII coloquio internacional sobre gestión universitaria en América del sur. Coloquio llevado a cabo en Universidad Nacional de Mar de Plata, Argentina.

Castro, G., y Noguera, Á. (2014). Depredación organizacional: las consecuencias del uso del poder en la organización. Estudios gerenciales, 30(132), 233-242.

Contreras, F., y Castro, G. (2013). Liderazgo, poder y movilización organizacional. Estudios gerenciales, 29(126), 72-76.

Cruz, L. (2013). El concepto de poder en la administración. Contaduría y administración, 58(4), 39-57.

De la Rosa, A. (2007). Poder, ambigüedad e institución: re-pensando la concepción tradicional de las relaciones de poder en el análisis organizacional. Administración y organizaciones, 11-29.

French, J y Raven B. (1982). Las bases del poder social. Barcelona: Estudios básicos de psicología social.

Foucault, M. (1988). El sujeto y el poder. Revista Mexicana de Sociología, 50(3), pp. 3-20.

Foucault, M. (1992). Microfísica del poder (Tercera ed.). Madrid: Ediciones Endymion.

Fuentes, D. (2014). Power relations: Ten faces of the influence of Ecopetrol and Modernization Project of the Barrancabermeja Refinery. Reflexión Politica, 16(32), 174-184.

García, Ó. (2009). El concepto de poder y su interpretación desde la perspectiva del poder en las organizaciones. Estudios Gerenciales, 25(110), 63-83. 
García, J. (2014). "Estar": fuente de poder genuino. IEEM Revista de Negocios, 8284.

Kadi, O. y Acevedo, A. (2014). Liderazgo ético frente a la diversidad cultural dentro de las organizaciones con régimen disciplinario. Económicas CUC, 35(2), 75-88.

Kim, P., Pinkley, R. y Fragale, A. (2005). Power dynamics in negotiation. Academy of Management Review, 30(4), 799-822.

Mariño, A. (2014). Las relaciones de poder y la comunicación en las organizaciones: una fuente de cambio. Ad-minister, (24), 118-141.

McClelland, D. (1989). Estudio de la motivación humana. Madrid: Narcea S.A.

Mintzberg, H. (1989). Diseño de organizaciones eficientes. México D.F.: El Ateneo.

Molina, M. (2012). La Comunicación Fuente de Poder del Líder en las Organizaciones que Aprenden. Revista Daena (International Journal of Good Conscience), 7(2), 56-60.

Murillo, G. (2009). Sociología de las organizaciones: Una perspectiva desde el poder y la autoridad para entender la cohesión social: el caso de la banca en Colombia. Pensamiento y Gestión, (26), 39-72.

Penaglia, F. (2012). La paradoja del poder: ¿elitización o empoderamiento colectivo? Polis, (32), 1-13.
Rodríguez, G. (2015). La función social de la dimensión emocional en el conflicto comunitario: entre la envidia, la desigualdad y las relaciones de poder. $E s$ tudios de Cultura Maya, 46(46), 167196.

Sanabria, R., Trujillo, M. y Guzmán, A. (2008). Poder y estrategia. Revista facultad de ciencias económicas, 16(2), 11-30.

Serna, M. (2013). Globalización, cambios en la estructura de poder y nuevas elites empresariales: Una mirada comparada de Uruguay. Revista de sociología y política, 21(46), 93-103+180-181.

Tirado, R. (2015). Enfoques teóricos y conceptos sobre el poder político empresarial. Revista Mexicana de ciencias políticas y sociales, 60(225), 311-339.

Vargas, V. (2014). Racionalidad en la prestación de servicios de salud. Algunas reflexiones. Económicas CUC, 35(1), 123-133.

Weber, M. (1997). Estructuras de poder. Buenos Aires.: La Pléyade.

Weber, M. (2007). Sociología del poder. Los tipos de dominación. Madrid: Alianza.

Weber, M. (2012). Economía y sociedad. Segunda reimpresión. México D.F.: Fondo de Cultura Económica.

Yela, J., e Hidalgo, C. (2010). El poder en Foucault: bases analíticas para el estudio de las organizaciones. Cuadernos de Administración, (44), 57-70. 